\title{
Open atrial transcatheter mitral valve replacement in patients with mitral annular calcification
}

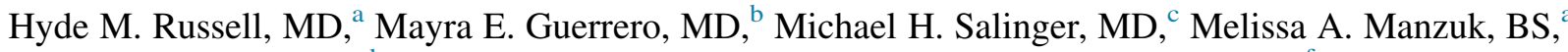
Amit K. Pursnani, MD, ${ }^{\mathrm{d}}$ Dee Wang, MD, ${ }^{\mathrm{e}}$ Hassan Nemeh, MD, ${ }^{\mathrm{e}}$ Rahul Sakhuja, MD, ${ }^{\mathrm{f}}$ Serguei Melnitchouk, MD, ${ }^{\mathrm{g}}$ Ashish Pershad, MD, ${ }_{\mathrm{h}}$ H. Kenith Fang, MD, ${ }^{\mathrm{h}}$ Sameh M. Said, MD, MBBCh, James Kauten, MD, ${ }^{\mathrm{j}}$ Gilbert H. L. Tang, MD, MSc, MBA, ${ }^{\mathrm{k}}$ Gabriel Aldea, MD, ${ }^{1}$ Ted E. Feldman, MD, ${ }^{\mathrm{d}}$ Vinnie N. Bapat, MD, MBBCh, ${ }^{\mathrm{m}}$ and Isaac M. George, $\mathrm{MD}^{\mathrm{m}}$

\section{ABSTRACT}

Background: Mitral valve replacement in the setting of severe mitral annular calcification remains a surgical challenge. Transcatheter mitral valve replacement (TMVR) using an aortic balloon-expandable transcatheter heart valve is emerging as a potential treatment option for high surgical risk patients. Transseptal, transapical, or transatrial access is not always feasible, so an understanding of alternative implantation techniques is important.

Objectives: The authors sought to present a step-by-step description of a contemporary transatrial TMVR technique using balloon-expandable aortic transcatheter heart valves. This procedure has evolved over time to address valve migration, left ventricular outflow tract obstruction, and paravalvular leak. The authors present a refined technique that has been associated with the most reproducible outcomes.

Methods: A step-by-step description of the TMVR technique and outcomes of 8 patients treated using this technique are described. Baseline patient clinical and echocardiographic characteristics and 30-day post-TMVR outcomes are presented.

Results: Eight patients underwent transatrial TMVR at a single institution. Five had previous cardiac surgery. Mean STS score was $8 \%$. Technical success by MVARC (Mitral Valve Academic Research Consortium) criteria was 100\%. There was zero in-hospital and 30-day mortality. Procedural success by MVARC criteria at 30 days was $100 \%$. Paravalvular leak immediately post-implant was none or trace in 6 and mild in 1 .

Conclusions: The technique described is reproducible and was associated with favorable outcomes in this early experience. It represents a useful technique for the treatment of mitral valve disease in the setting of severe annular calcification. A structured and defined implantation technique is critical to investigators as this field evolves. (J Thorac Cardiovasc Surg 2019;157:907-16)

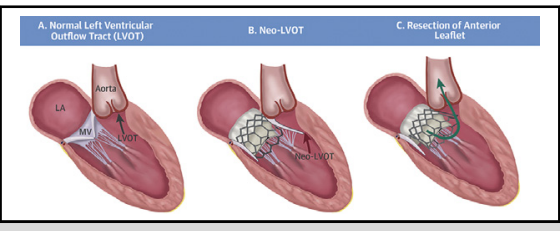

Prevention of left ventricular outflow tract obstruction

Central Message

Patients with severe MAC undergoing either surgical MVR or percutaneous TMVI face risks of complications including embolization, paravalvular leak, and left ventricular outflow obstruction. Early experience with a hybrid surgical approach direct trans-atrial TMVI addresses these issues.

\section{Perspective}

Longer-term follow-up studies in larger cohorts of patients with severe MAC are needed to establish optimum procedural technique and evaluate the safety and efficacy of the hybrid trans-atrial approach.

See Commentaries on pages 917 and 919.

\footnotetext{
From the Divisions of a Cardiovascular Surgery and ${ }^{\mathrm{d}}$ Cardiology, NorthShore University HealthSystem, Evanston, Ill; ' Department of Cardiovascular Medicine, Mayo Clinic Hospital, Rochester, Minn; ${ }^{\mathrm{c} D}$ Division of Cardiology and Cardiovascular Surgery, Froedtert/Medical College of Wisconsin, Milwaukee, Wis; ${ }^{\mathrm{e} C e n t e r}$ for Structural Heart Disease, Henry Ford Hospital, Detroit, Mich; Divisions of ${ }^{\mathrm{f}}$ Cardiology and ${ }^{\mathrm{g}}$ Cardiac Surgery, Massachusetts General Hospital, Boston, Mass; ${ }^{\mathrm{h}}$ BannerUniversity Medicine Heart Institute, Phoenix, Ariz; ${ }^{i}$ Department of Cardiovascular Surgery, Mayo Clinic, Rochester, Minn; ${ }^{j}$ Marcus Heart Valve Center, Piedmont Heart Institute, Atlanta, Ga; ${ }^{\mathrm{k}}$ Department of Cardiovascular Surgery, Mount Sinai Medical Center, New York, NY; ${ }^{\mathrm{D}}$ Division of Cardiothoracic Surgery, University of Washington, Seattle, Wash; and the ${ }^{\mathrm{m}}$ Division of Vascular, Thoracic and Cardiac Surgery, New York Presbyterian Hospital-Columbia University Medical Center, New York, NY.
}

This article is co-published in the Journal of the American College of Cardiology. The American College of Cardiology requests that this document be cited as follows: Russell HM, Guerrero ME, Salinger MH, et al. Open atrial transcatheter mitral valve replacement in patients with mitral annular calcification. J Am Coll Cardiol. 2018;72:1437-48.

Received for publication May 24, 2018; accepted for publication July 2, 2018.

Address for reprints: Hyde M. Russell, MD, Division of Cardiovascular Surgery,

NorthShore University Health System, 2650 Ridge, Walgreen Building, 3rd Floor

Evanston, IL 60201 (E-mail: hrussell@ northshore.org). $0022-5223 / \$ 36.00$

Copyright (C) 2018 by the American College of Cardiology Foundation https://doi.org/10.1016/j.jtcvs.2018.09.003 


\section{Abbreviations and Acronyms \\ $\mathrm{CT}=$ computed topography \\ ECG = electrocardiogram \\ $\mathrm{LV}=$ left ventricle/ventricular \\ LVOT $=$ left ventricular outflow tract \\ MAC $=$ mitral annular calcification \\ MVR = mitral valve replacement \\ PVL = paravalvular leak \\ TAVR $=$ transcatheter aortic valve replacement \\ $\mathrm{THV}=$ transcatheter heart valve \\ $\mathrm{TMVR}=$ transcatheter mitral valve replacement}

Video clip is available online.

The treatment of patients requiring mitral valve replacement (MVR) in the setting of severe mitral annular calcification (MAC) remains a surgical challenge. These patients tend to be older with multiple comorbidities. ${ }^{1}$ In addition, the surgical procedure itself is fraught with several well-described complications, including patient-prosthesis mismatch secondary to using an undersized valve, paravalvular leak (PVL), left circumflex coronary artery injury, and atrioventricular groove rupture. ${ }^{2}$ Because of these complications and the risks associated with comorbidities, long-term outcomes of this operation have historically been poor, and many patients with the condition are not offered mitral valve surgery.

Multiple surgical strategies have been employed over time to deal with this challenging problem. ${ }^{3-24}$ These include complete resection of the entire calcium bar with annular reconstruction, atrial sliding plasty, left atrial to left ventricular (LV) apical conduit, ${ }^{25}$ and anterior leaflet foldover. Attempts to spare the calcium and debride only what is necessary in order to implant the valve have also been used and advocated. Despite the numerous approaches to this problem, complications remain frequent, and no one strategy has gained widespread acceptance with reproducible outcomes.

Use of a balloon-expandable transcatheter heart valve (THV) has the potential to overcome many of the limitations of traditional surgical approaches in patients with heavy or prohibitive MAC. However, limitations due to positioning, anchoring, PVL, and LV outflow tract (LVOT) obstruction exist. Transapical and transseptal techniques, although less invasive than open surgery, may not be feasible due to inadequate anchoring in unfavorable MAC patterns, or may fail to address potential LVOT obstruction or potential PVL. These limitations of transcatheter THV implantation limit universal adoption of purely catheterbased delivery for patients with severe MAC.
Use of a balloon-expandable prosthesis via a direct transatrial approach has been previously described using various techniques. ${ }^{26-32}$ The transatrial approach allows for the potential to address many of the limitations of alternative surgical or transcatheter therapies in patients with severe MAC. The first successful use of a Sapien XT prosthesis (Edwards Lifesciences, Irvine, Calif) in a patient with severe MAC undergoing planned MVR was reported in 2012. ${ }^{33}$ This was followed by other reports for bailout in complex settings. ${ }^{34}$ In 2016, the first successful planned transatrial use of the Sapien XT in MAC was reported, setting the stage for others to optimize this approach. ${ }^{35}$ Outcomes, however, have remained inconsistent and suboptimal as reported in large global registries, ${ }^{36}$ smaller U.S. national registries, ${ }^{37}$ and within the recently presented MITRAL (Mitral Implantation of Transcatheter Valves) trial. $^{38}$

We have recently modified an open transatrial technique utilizing the Sapien 3 (S3) THV that allows for accurate valve positioning, prevents valve migration, appears to prevent acute PVL, and allows for mitigation of potential LVOT obstruction. We feel this technique using a balloonexpandable prosthesis represents an iterative evolution based on numerous cases, simplifies the operation, and facilitates better and more predictable results. Our procedural steps and early results are described herein.

\section{METHODS}

\section{Pre-Procedural Planning}

As with other transcatheter valve therapies, pre-procedural planning is critical to obtain optimal results. All patients undergo a standard workup with transthoracic and transesophageal echocardiography, coronary angiography, and a cardiac gated multidetector computed tomography (CT) scan. Important comorbidities, such as concomitant valvular disease, lung disease, pulmonary hypertension renal disease, frailty/surgical fitness, and right ventricular function, are carefully assessed according to standard procedures, and all patients are reviewed by the multidisciplinary heart team.

\section{Cardiac CT: Image Acquisition Technique}

Similar to transcatheter aortic valve replacement (TAVR), CT imaging has proven to be the most reliable imaging tool for prosthesis size selection and is of particular importance in assessment of the LVOT pre-operatively. Our use of cardiac CT imaging to assess and plan mitral valve-in-valve replacement procedures has been documented previously, ${ }^{39,40}$ and more recently, an initial report of 3-dimensional prototyping for procedural simulation of transcatheter mitral valve replacement in MAC has been reported. ${ }^{41}$

CT in preparation for transcatheter mitral valve replacement (TMVR) should include the imaging of the entire heart. Overall, the protocol is similar to CT protocols for TAVR, ${ }^{42}$ with adjustments for mitral valve analysis. ${ }^{43}$ The goal is to provide sufficient contrast enhancement of the left atrium and LV to display relevant mitral valve anatomy, and to provide these data throughout the cardiac cycle. Contrast opacification is needed for assessment of LVOT obstruction risk as detailed later in the text. Beta-blockers for heart rate slowing can be considered, particularly for patients with fast heart rates ( $>80$ beats/min) or arrhythmias (frequent atrial ectopy), although as discussed later in the text, advanced techniques for 
image reconstruction can help to avoid the use of beta-blockers. Although the image acquisition protocol will depend on the institution's scanner, in general, imaging of the heart should be performed using a helical acquisition with retrospective electrocardiogram (ECG) gating. Dose modulation is turned off to allow for comprehensive imaging of mitral valve anatomy across the entire cardiac cycle. At our institution, the CT protocol is as follows using a Siemens 128-slice dual source FLASH scanner, although this protocol is applicable to all 64-slice scanners or new-generation scanners:

1. Scout image from lung apices to diaphragm.

2. Noncontrast CT of chest from tracheal bifurcation to below the heart contour. We use the high-pitch helical (FLASH) mode for this noncontrast acquisition.

3. Test bolus for timing of contrast using flow rate of 4 to $5 \mathrm{~mL} / \mathrm{s}$. Scan delay is determined using region of interest drawn in the left atrium. The amount of contrast used for test bolus is typically 10 to $15 \mathrm{~mL}$.

4. Retrospective ECG-gated scan from aortic root to below the apical contour of heart. Tube voltage is typically $120 \mathrm{kvp}$ to reduce beamhardening artifact related to dense MAC. Tube current is scanner specific and can be adjusted to the lowest setting allowing acceptable image noise. The usual amount of contrast used ranges between 65 and $85 \mathrm{~mL}$.

\section{CT: Image Reconstruction}

A multiphase $(0 \%$ to $95 \%)$ image reconstruction with slice thickness of $<1.25 \mathrm{~mm}$ should be obtained at $5 \%$ to $10 \%$ increments of the cardiac cycle and include the entire heart. Sharper convolution kernels can be used to reduce local blooming artifact from calcium. This reconstruction can be utilized for the post-processing steps detailed in the following text. For patients with arrhythmias such as atrial fibrillation, multiple approaches can be used to ensure optimal image quality. First, ECG editing can be used at the scanner to remove ectopic beats or those with very short R-R intervals. Second, absolute delay reconstruction can be used where a specified absolute delay after the R-wave can be reconstructed (ie, 0 to $400 \mathrm{~ms}$ ). This is preferable in patients with irregular heart rhythms because the duration of systole (first few hundred milliseconds from the R-wave) does not change significantly with varying R-R intervals.

\section{Cardiac CT Analysis}

Sizing. Analysis of the cardiac CT is performed using commercially available software (3Mensio Structural Heart Mitral Workflow version 8.1; Pie Medical Imaging, Maastricht, The Netherlands). All cardiac phases are evaluated as the annulus size may be different in systole and diastole. The best systolic phase is chosen to allow more accurate evaluation of the risk of LVOT obstruction post-TMVR implant, which is greater in systole.

The mitral annular diameters and area in the diastolic phase are defined using the systematic method in the 3Mensio software version 8.1 (Pie Medical Imaging, Maastricht, The Netherlands) (Figure 1,A). Unlike the transfemoral transseptal approach where the best fluoroscopy deployment angles are determined using the 3Mensio software, fluoroscopy is not

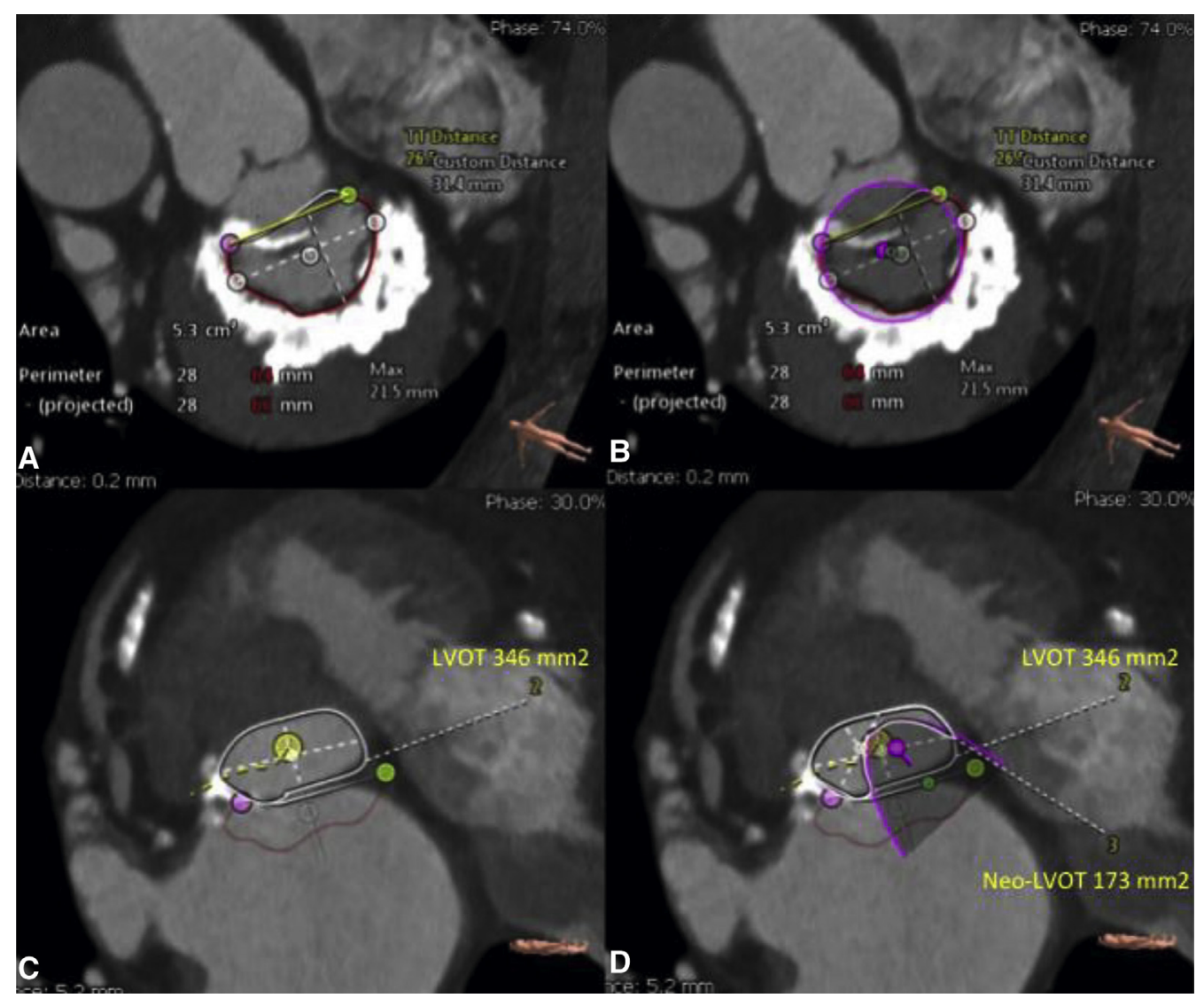

FIGURE 1. CT scan pre-operative planning. Cardiac computed tomography-based measurement of the calcified mitral annulus using $3 \mathrm{Mensio}$ Structural Heart Mitral Workflow version 8.1 (Pie Medical Imaging, Maastricht, The Netherlands). A, The mitral annular diameter and area in the diastolic phase are defined. B, A simulation of the THV is overlaid with the patient's images to assess position and sizing. C, Baseline LVOT is measured. D, Expected "neoLVOT" is measured to predict risk for significant LVOT obstruction. LVOT, Left ventricular outflow tract. 
TABLE 1. Open transatrial balloon-expandable TAVR prosthesis in severe MAC: Procedural steps summary

Transatrial approach to sapien 3 mitral valve in severe mitral annular calcification

1. Standard sternotomy or right thoracotomy

2. Prepare Edwards Sapien 3 transcatheter valve

a. Cut felt (Bard PTFE Felt $2.5 \mathrm{~cm} \times 15.2 \mathrm{~cm}$ Ref 007976) to a strip $0.75-\mathrm{cm}$ wide for $26-\mathrm{mm}$ valve or $1-\mathrm{cm}$ wide for $29-\mathrm{mm}$ valve

b. Sew felt cuff around the sealing skirt with running 5-0 Prolene; trim excess length

c. Place interrupted 5-0 Prolene sutures at the top of the felt strip at each commissure to future secure felt

d. Place 3 guiding sutures (3-0 Ethibond, double armed) at the commissures of the valve $\left(120^{\circ}\right.$ apart)

e. Place valve in saline to be crimped later

3. Aortic cannulation

4. Bicaval venous cannulation

5. Standard diastolic arrest with antegrade cardioplegia

6. Open left atrium in Waterston's groove

7. Place self-retaining mitral valve retractor

8. Excise anterior leaflet and all supporting chords

9. Leave posterior leaflet intact; consider transecting chords

10. Pledgeted 2-0 Ethibond suture around circumference of mitral valve annulus with pledgets placed on the atrial side

a. Posterior leaflet region: place pledgeted sutures through atrial tissue just outside the mitral annulus to allow for a hefty rim of tissue for valve to snug up against

11. Mark commissures on native mitral annulus using Edwards Aortic Valve sizer (which has appropriate markings) to place marks $120^{\circ}$ apart

a. Place marks so 2 commissures would subtend the LVOT

12. Crimp previously prepared valve

13. Bring prepared valve onto field

14. Place guiding sutures through marks on the native mitral annulus and place snare

15. Lower valve into position and slowly snare guiding sutures as the valve balloon is inflated

\section{Secure 3 guiding sutures with Cor-Knots}

17. Pass previously placed pledgeted 2-0 Ethibond sutures through the stent frame and sealing skirt and secure with Cor-Knots or hand tie

18. De-air and close left atrium

19. De-air, remove cross clamp, re-perfuse, and wean from bypass

20. Assess valve function, presence of paravalvular leak, and presence of LVOT obstruction using TEE

LVOT, Left ventricular outflow tract; TEE, transesophageal echocardiography.

used when the implantation is done under direct visualization using the transatrial approach. Additionally, the orientation of the heart shifts dramatically once the patient is on bypass, making pre-operative fluoroscopy angles of no value during THV deployment within a flaccid heart.

The THV size is selected on the basis of the inner diameter and area measurements obtained from the $\mathrm{CT}$ analysis and the THV sizing charts as in TAVR. In current practice, the type of valve used for this application is the Sapien 3 valve. A simulation of the selected THV size can be overlaid with the patient's images to assess position and sizing inside the mitral annulus (Figure 1, B). The typical target for simulated valve positioning is to have approximately $80 \%$ of the THV stent frame in the LV and $20 \%$ in the left atrium.

Neo-LVOT measurement. Once the virtual valve is embedded in the CT image, its appearance is evaluated in the deployed position. Once the landing zone of the THV is defined, the distance from the interventricular septum to the frame of the THV is measured and the LVOT area at this precise level is assessed (the closest point of the THV to the septum). The virtual valve is removed to make the measurement of the baseline LVOT at this level (Figure 1,C). The measurement is repeated with the virtual THV in place to determine the remaining area in the LVOT at this level. This is the expected "neo-LVOT" area after TMVR (Figure 1,D). The cutoff neoLVOT area at which there is significant risk for LVOT obstruction after TMVR has not been fully defined at this time. On the basis of prior studies, a neo-LVOT area of $250 \mathrm{~mm}^{2}$ or more should have low risk of LVOT obstruction even with transseptal and apical implant approaches. ${ }^{44,45}$ It is important to make these measurements in systole when the LVOT area is smallest. Usually the $45 \%$ systolic phase is most appropriate. It should be recognized when reviewing the CT data that unlike the femoral transseptal approach, the open transatrial approach allows for resection of a significant portion of the anterior mitral leaflet at the time of the procedure. This optimizes the neo-LVOT by allowing the ventricular row of THV cells to remain open and unobstructed by native mitral leaflet during systole. Thus, the actual LVOT flow with a transatrial approach is a combination of both the measured neo-LVOT flow plus THV flow through the cells. Consequently, the acceptable range for neo-LVOT may likely be much lower using the open transatrial approach, and levels as low as $150 \mathrm{~mm}^{2}$ have been safely treated. The lower limits of safe neo-LVOT area are not established. Finally, the option of a surgical myectomy should be considered if there is concern about LVOT gradient or flow despite anterior mitral leaflet resection. This has been performed with success through either a transaortic or transmitral approach.

\section{Pre-Procedural Medical Optimization}

Optimization of hemodynamics is an important component of our preprocedural planning. We often have the patients evaluated and treated by our heart failure service during the days and weeks before the procedure so that the patient arrives in the operating room as medically optimized as possible.

\section{Surgical Technique}

Please consult text and figures, as well as the step-by-step outline in Table 1, and Videos 1 and 2.

Patients who have not undergone previous surgery are generally approached with a routine median sternotomy. The technique is also applicable to a right thoracotomy exposure via standard or minimally invasive approaches.

A back table is set up with all necessary instruments to prepare the Sapien 3 THV (Figure 2, A). Based on pre-operative CT and echocardiographic data, the appropriately sized Sapien S3 valve, most commonly $29 \mathrm{~mm}$, can be opened and placed in a bowl of saline at room temperature at this point. Before commencing cardiopulmonary bypass, we prepare the valve with a Teflon felt strip and guiding sutures. The felt strip (polytetrafluoroethylene [PTFE] Felt $2.5 \mathrm{~cm} \times 15.2$-cm BARD Reference number 007976; Bard Peripheral Vascular, Tempe, Ariz) is cut to width $(0.75 \mathrm{~cm}$ for a 26-mm valve; $1 \mathrm{~cm}$ for a 29-mm valve) (Figure 2, $B$ ) and anastomosed to the bottom (atrial side) of the stent frame with a running 5-0 Prolene (polypropylene) suture (Ethicon, Somerville, NJ) (Figure 2, C). The felt strip is anchored at each commissure with an interrupted 5-0 Prolene suture (Figure 2,D). These 3 sutures keep the felt strip from sliding off during crimping. Finally, we place 3 guiding sutures of 3-0 Ethibond at each 


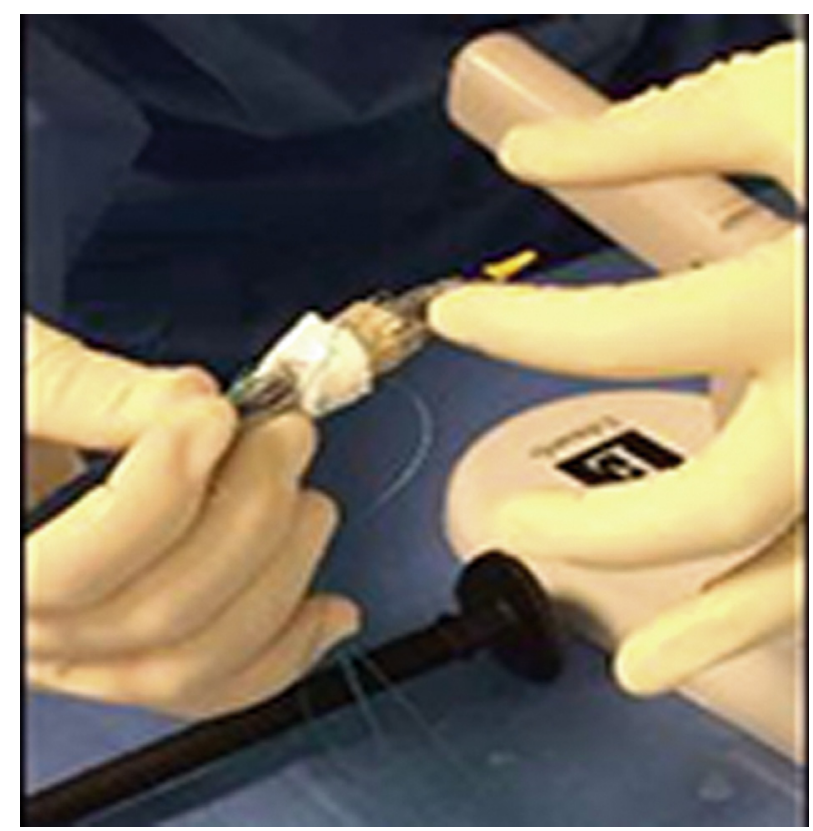

VIDEO 1. This video is an example of one of our cases using the described technique. Video available at: https://www.jtcvs.org/article/S00225223(18)32349-3/fulltext.

commissure (Figure 2, E). This suture is best performed by passing the needle from outside in, grasping the needle, and then passing it inside out around one of the stent frame bars, paying attention to avoid damage to the prosthetic leaflets. These sutures are left long with both needles remaining. The valve is replaced into the bowl of saline (Figure 2,F) until we are ready for it at the field, at which time it will be crimped (Figure 3).

The mitral valve is exposed via a left atriotomy, and a valve analysis is performed. The anterior leaflet is excised, leaving a standard rim of tissue at the aortomitral curtain that will be used to place the pledgeted anchoring sutures (Figure 4, A). Because the anterior leaflet and subvalvular apparatus is often scarred, calcified, and foreshortened, we have not made an attempt to spare anterior cords in our experience thus far. Occasionally, the papillary muscle may have to be partially resected to avoid interaction with the Sapien 3 THV. The posterior leaflet and annulus are left intact. No attempt is made to debride the calcium.

Pledgeted valve sutures are placed through the aortomitral curtain anteriorly and around the posterior annulus using a rim of left atrial tissue because the annular tissue is often too calcified to penetrate with needles (Figure 4, B). This avoids having to pass the needles through the bulky calcium. The septum and LVOT are evaluated, and a decision of where the prosthetic commissures should lie is performed. A dental mirror can be helpful in determining position. A standard valve sizer with $120^{\circ}$ markings is used to mark the intended placement of the 3 guiding sutures (Figure 4, C). These sutures will ultimately ensure that the prosthetic commissures are oriented such that they subtend the LVOT.

The Edwards valve is then crimped on the back table onto the Certitude balloon delivery system (Edwards Lifesciences) with the proper orientation for the "skirt" to be atrial and the open cell LV outflow of the THV closest to the nose cone of the delivery system. This is the same orientation as the usual transapical TAVR configuration. Careful, slow crimping should allow for a symmetrical result despite the added felt inside the crimper. Of note, the sleeve that is traditionally used when crimping a standard Sapien 3 valve is not necessary, and the valve does not need to be fully crimped. The inflation syringe is filled with the nominal volume of saline. It not uncommon to add an additional 3 to $5 \mathrm{ml}$ of volume to the inflation device based on the CT sizing data obtained pre-operatively. The delivery system

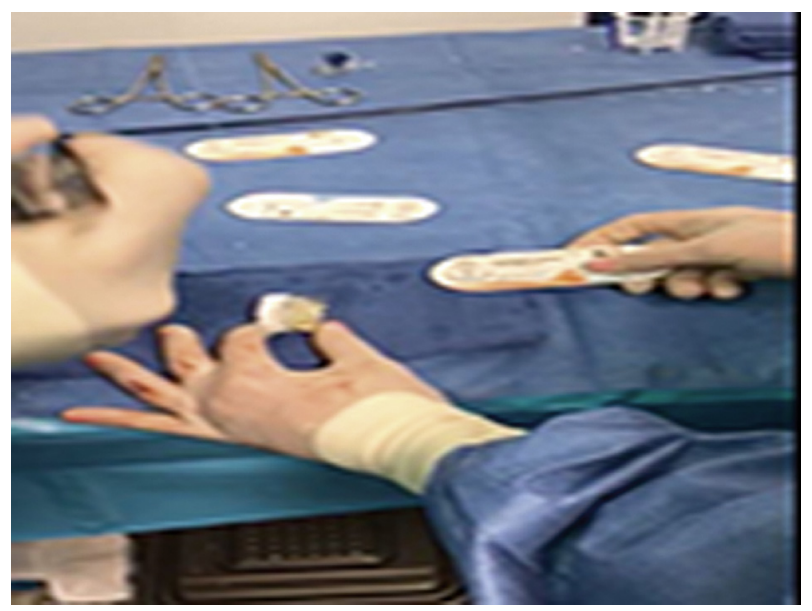

VIDEO 2. This video shows step-by-step instructions of the valve preparation using the additional felt strip and the guiding sutures as well as crimping. Video available at: https://www.jtcvs.org/article/S00225223(18)32349-3/fulltext.

and valve are then brought to the surgical field, taking care not to entangle the 3 guiding sutures. A standard 0.035 -inch $\mathrm{J}$ wire is placed through the balloon catheter and down into the LV through the mitral valve and between the papillary muscles. We believe the use of the $\mathrm{J}$ wire helps protect the ventricle from the nosecone of the delivery system (Figure $4, D$ ). The guiding sutures are then passed through the annulus at the previously marked sites. The balloon and valve are lowered into position. The 3 guiding sutures are snared gently. The balloon is then slowly inflated to approximately $50 \%$, and the snares are gradually tightened as the valve expands toward the annulus. The snared guiding sutures are intended to align the valve in the correct orientation as well as set the proper height of the valve respective to the mitral annulus (Figure $4, E$ ). The balloon is then fully inflated, expanding the valve completely. The balloon is deflated, and the delivery system removed. The 3 guiding sutures are then secured either by hand or using Cor-Knots (LSI Solutions, Victor, NY). Finally, the pledgeted sutures encircling the base of mitral leaflets are passed through the felt ring and stent frame. They are secured on the inside of the valve frame (Figure 4,F). These sutures are critically important in preventing PVL, and the seal should appear uniform around the circumference of the valve. The valve is then tested with saline irrigation to ensure all 3 leaflets coapt and that there is no PVL leak. The left atrium is then closed, the heart is deaired, and the patient is weaned and separated from cardiopulmonary bypass in standard fashion.

A video demonstration of our technique may be found online. ${ }^{46}$

\section{Technical Variations}

1. Balloon sizing after leaflet resection has been performed to assist in valve sizing. An Edwards delivery system balloon of the proposed size is used before opening the valve itself. If the balloon fits tightly at the nominal volume, there is assurance that the correct-sized valve has been used. If the balloon slides loosely within the annulus or is significantly underexpanded, consideration for a larger or smaller valve size should be given. Additionally, adjusting the saline volume used for valve inflation to ensure optimal seal can be considered. If balloon sizing is performed, preparation of the valve occurs after valve examination and exposure. This technique can be helpful when there is concern over sizing or when there is a question that even the largest THV available $(29 \mathrm{~mm})$ will be too small to fill the mitral orifice. We are generally able to select the valve size based on careful CT analysis.

2. The procedure may be completed without the use of guiding sutures, but they may serve to aid valve positioning and valve orientation, and 

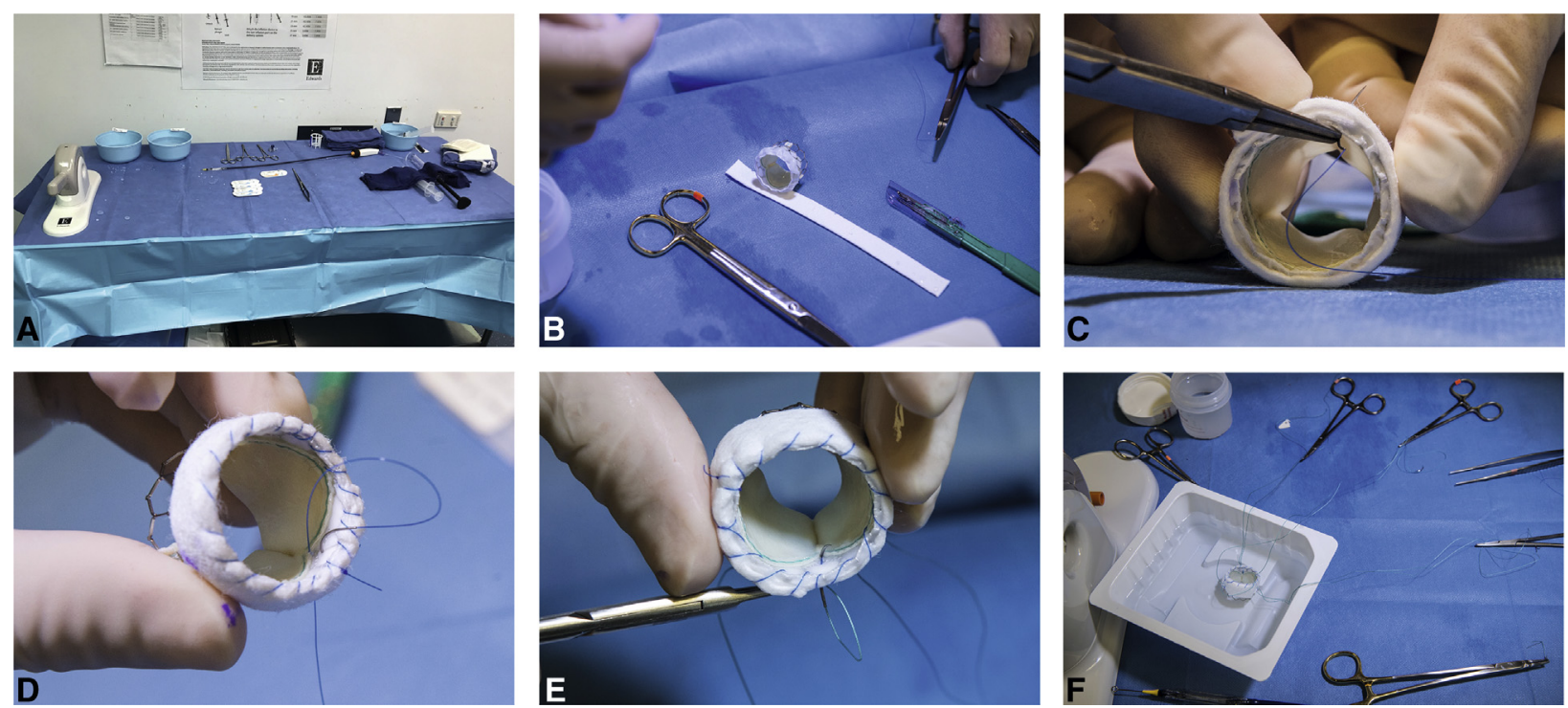

FIGURE 2. Valve preparation. A, Back table setup with all necessary instruments for valve preparation. B, Felt strip (PTFE) cut to appropriate dimensions: 1-cm width for 29-mm valve, $0.7-\mathrm{cm}$ width for 26-mm valve. C, Anastomosis of felt strip to the bottom of the stent frame using a running 5-0 Prolene suture. $\mathrm{D}$, Anchoring of felt strip at each commissure using an interrupted 5-0 Prolene suture. Suture is kept away from the leaflets and below the green manufacturer suture. E, Placement of 3 guiding sutures using 3-0 Ethibond at each commissure. Needles are passed outside in, grasped, and then passed inside out around the stent frame below green manufacturer suture line. Sutures are left long with needles remaining. F, Place valve into a bowl of room temperature saline where it remains until it is crimped before bringing up to the field. See Video 2.

provide additional protection against valve migration. Alternatively, the commissure can be marked with a pen before crimping, and the valve is manually positioned without guiding sutures. Orientation of the THV commissures is important if LVOT obstruction is to be minimized.

3. Commissural plication using a figure-of- 8 Prolene suture may be performed. Given the D-shape of the native mitral annulus, this may help to create a more circular shape and potentially improve the seal and reduce PVL.

4. Some surgeons have tried to minimize or eliminate the use of the annular sutures. We strongly feel that the time spent placing multiple pledgeted sutures encircling the native leaflet bases is important to minimize PVL and is time well spent.

5. Crimping the valve only $50 \%$ to allow better visualization of the prosthesis inside the annulus has been proposed, followed by placing and inflating the balloon once the valve position is confirmed. This may be particularly helpful when the LV is small and short, and the nose cone abuts the apex, preventing satisfactory seating of the valve at the correct height.

6. Resection of all subvalvular tissue, chordae, and even papillary muscles may sometimes be necessary to allow full expansion of the Sa- pien 3 valve or prevent interaction with the valve stent frame. In addition, the valve can be crimped closer to the nose cone side of the balloon to reduce the length of the delivery apparatus inside the LV cavity.

7. Some operators have performed septal myectomy before valve implantation through either the aortic valve or the mitral valve to decrease the risk of LVOT obstruction in patients with very severe septal hypertrophy. This has not been our routine practice because it adds to the complexity of the case and engenders myectomy-related risks. To avoid the need for myectomy in these cases, we would consider alcohol septal ablation several weeks before the planned THV implant procedure.

\section{Post-Op Care}

Similar to conventional bioprosthetic mitral valve replacement, all patients receive lifelong aspirin and a minimum of 3 months of warfarin with a target international normalized ratio of 2.0 to 3.0. Anticoagulation is particularly important in this setting given the stent frame, prosthesis, and the felt strip that may be a nidus for thrombus formation. There have been reports of valve thrombosis of balloon-expandable
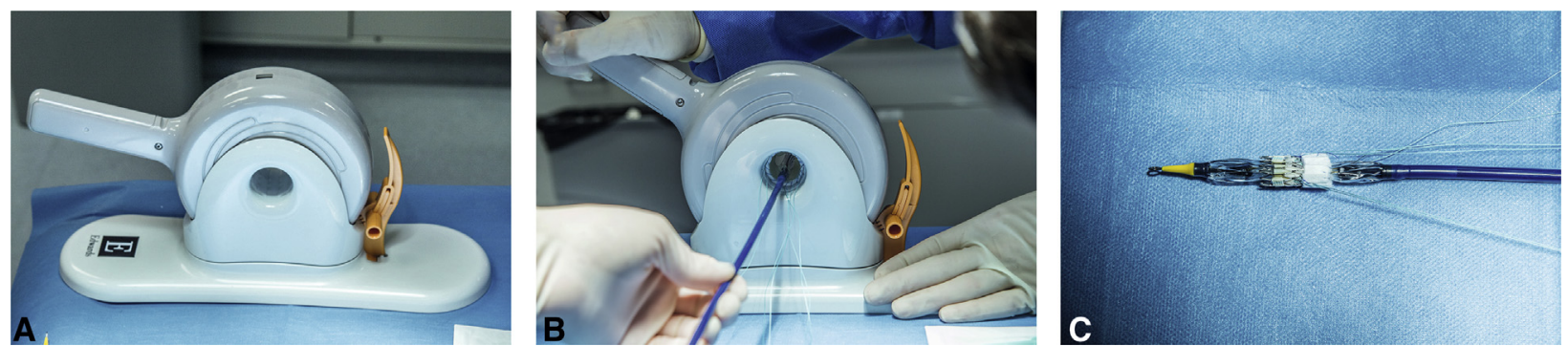

FIGURE 3. Valve crimping. A, Prepared valve prior to crimping with 3 Ethibond sutures left long with needles attached. B, Crimping of the Edwards Sapien 3 valve on a balloon delivery system is completed on the back table before bringing up to the surgical field. C, Proper orientation for the "skirt" to be atrial and the open cells toward the left ventricular outflow of the THV closest to the nosecone of the delivery system. 

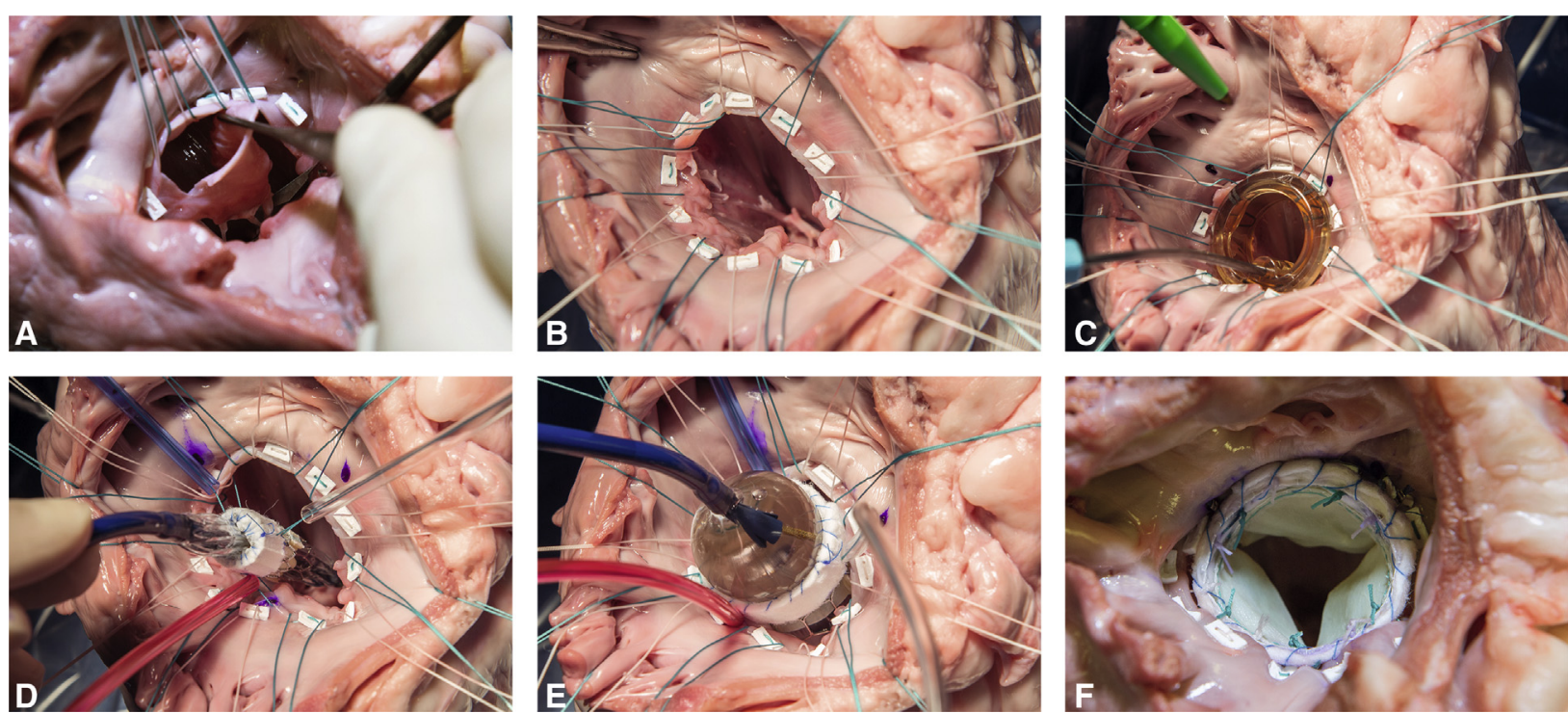

FIGURE 4. Valve implantation. A, Anterior leaflet is resected leaving a rim of atrial tissue. Pledgeted 2-0 Ethibond sutures are placed through this rim of tissue. B, After resection of the anterior leaflet, pledgeted valve sutures are placed through aortomitral curtain anteriorly and around the posterior annulus using a rim of left atrial tissue. Sutures do not need to be passed through the posterior annular calcium. C, Using a standard valve sizer with $120^{\circ}$ markings, mark where 3 guiding sutures will be placed. The anterior markers will subtend the LVOT. D, The balloon delivery system and valve are brought up to the table with a standard 0.035 -inch J wire placed through the delivery system into the left ventricle. The guiding sutures are placed through the annulus at the previously marked positions and then snared (red, blue, clear snares in this picture). E, The balloon is inflated as the 3 guiding sutures are gently snared. F, Three guiding sutures are secured. Pledgeted sutures encircling the base of the mitral leaflets are passed through the felt strip and valve frame, and then secured. All photos were taken from simulation laboratory using bovine calf hearts. See Video 1.

aortic valves in the mitral position. ${ }^{47,48}$ Direct oral anticoagulants are not recommended.

Patients are typically followed at 30 days with a transthoracic echo and $\mathrm{CT}$ to assess valve positioning and LVOT anatomy. Further clinical and echocardiographic follow-up is performed at 6 months and 1 year, and then yearly afterwards.

\section{RESULTS}

The technique for transatrial TMVR described in this paper was used at 1 institution in a nonrandomized fashion at the operator's discretion in 8 patients. Five of the 8 patients had undergone previous cardiac surgery. Mean STS score was $8 \%$. One patient underwent a concomitant aortic valve replacement, and 2 underwent concomitant tricuspid valve repair. Operative details are listed in Table 2.

Using endpoints defined by the Mitral Valve Academic Research Consortium, ${ }^{49}$ the THV was successfully implanted with $100 \%$ technical success in all cases. Procedural success at 30 days was also $100 \%{ }^{49}$ PVL immediately post-implantation was none or trace in 6 patients and mild in 1 . There were no cases of moderate or severe PVL. One patient with mild PVL post-TMVR developed hemolysis 6 months post-TMVR that was successfully treated with percutaneous closure using a vascular plug. There were no procedural major complications, including clinically significant LVOT obstruction, annular rupture, valve embolization, or migration. The mean length of stay has been 7.9 days following surgery. There were no in-hospital or 30-day mortalities. No patient had a stroke.
One patient (\#5) died at home 7 months post-operatively; all other patients are alive.

\section{DISCUSSION}

Conventional surgical valve replacement techniques for dealing with the calcium inherent in this disease can be generally be categorized in terms of "resect or respect." Resecting the entire calcium bar down to the adventitial fat of the atrioventricular groove places the patient at risk for both atrioventricular groove disruption and circumflex artery injury. The advantage of calcium resection is the ability to implant a larger prosthesis and generally have a low PVL rate because of tissue compliance. In some cases, annular debridement will allow for valve repair. The technique described in this paper does not debride the calcium but still allows for a large prosthesis implantation because of the expandable valve technology. It is still theoretically possible to rupture the annulus if too large a prosthesis is selected, just as this complication has been seen in the TAVR population. Pre-operative imaging analysis to select the optimal size of the valve therefore remains critical. We therefore do not consider this a "bailout" option when standard valve replacement is discovered to be difficult, but rather a deliberately planned operation.

Early attempts at using transcatheter valves to replace the mitral valve in the setting of MAC either via the transseptal, transapical or transatrial approach have been complicated by the presence of significant PVL in many cases. The 
TABLE 2. Patient characteristics and outcomes

\begin{tabular}{|c|c|c|c|c|c|c|c|c|c|c|c|c|c|c|c|c|}
\hline $\begin{array}{c}\text { Patient } \\
\#\end{array}$ & Date & $\begin{array}{c}\text { Age, } \\
\text { yrs }\end{array}$ & Sex & $\begin{array}{c}\text { STS, } \\
\%\end{array}$ & $\begin{array}{c}\text { Prior } \\
\text { cardiac } \\
\text { surgery }\end{array}$ & $\begin{array}{c}\text { LVEF, } \\
\%\end{array}$ & $\begin{array}{c}\text { Baseline } \\
\text { LVOT, } \\
\text { mm }^{2}\end{array}$ & $\begin{array}{c}\text { Predicted } \\
\text { LVOT, } \\
\text { mm }^{2}\end{array}$ & $\begin{array}{l}\text { Valve } \\
\text { size, } \\
\text { mm }\end{array}$ & $\begin{array}{l}\text { Concurrent } \\
\text { TV repair }\end{array}$ & $\begin{array}{c}\text { CPB } \\
\text { time, } \\
\text { min }\end{array}$ & $\begin{array}{c}\text { Cross } \\
\text { clamp } \\
\text { time, } \\
\text { min }\end{array}$ & $\begin{array}{c}\text { Technical } \\
\text { success }\end{array}$ & $\begin{array}{l}\text { ICU, } \\
\text { days }\end{array}$ & $\begin{array}{c}\text { Ventilator, } \\
\mathbf{h}\end{array}$ & $\begin{array}{c}\text { Blood } \\
\text { transfusion, } \\
\mathbf{U}\end{array}$ \\
\hline 1 & $3 / 3 / 17$ & 65 & $\mathrm{M}$ & 12 & Yes & 65 & 357 & 120 & 29 & No & 103 & 70 & Yes & 3 & 9 & 3 \\
\hline 2 & $6 / 8 / 17$ & 78 & $\mathrm{~F}$ & 6.3 & Yes & 66 & 400 & 213 & 29 & Yes & 112 & 88 & Yes & 2 & 9 & 0 \\
\hline 3 & $7 / 3 / 17$ & 74 & M & 8 & Yes & 65 & 512 & 292 & 29 & No & 185 & 90 & Yes & 3 & 7 & 4 \\
\hline 4 & $10 / 5 / 17$ & 87 & $\mathrm{~F}$ & 8.5 & No & 60 & 352 & 109 & 29 & No & 89 & 55 & Yes & 3 & 6 & 0 \\
\hline 5 & $10 / 11 / 17$ & 80 & M & 13 & Yes & 37 & 517 & 299 & 29 & No & 151 & $\mathrm{n} / \mathrm{a}^{*}$ & Yes & 2 & 10 & 0 \\
\hline 6 & $12 / 14 / 17$ & 77 & M & 5.8 & Yes & 65 & 472 & 335 & 29 & No & 187 & $\mathrm{n} / \mathrm{a}^{*}$ & Yes & 3 & 10 & 0 \\
\hline 7 & $1 / 25 / 18$ & 75 & $\mathrm{~F}$ & 8 & No & 65 & 249 & 53 & 26 & Yes & 151 & 104 & Yes & 2 & 5 & 1 \\
\hline 8 & $4 / 12 / 18$ & 69 & $\mathrm{~F}$ & 3 & No & 69 & 293 & 37 & 26 & No & 180 & 160 & Yes & 2 & 13 & 0 \\
\hline
\end{tabular}

$S T S$, Society of Thoracic Surgeons score; $L V E F$, left ventricular ejection fraction; $L V O T$, left ventricular outflow tract; $T V$, tricuspid valve; $C P B$, cardiopulmonary bypass; $I C U$, intensive care unit; $M$, male; $F$, female. *Procedure was done under fibrillatory arrest.

current technique described appears to reliably allow for successful valve replacement in even the most challenging circumstances without significant PVL.

The potential for LVOT obstruction is dealt with in both the pre-operative planning phase and during the surgical procedure itself. The importance of the pre-operative CT scan in analyzing the "neo-LVOT" following implantation of the various size prostheses cannot be over emphasized. The ability to surgically resect a large portion of the anterior mitral leaflet via the transatrial approach significantly mitigates the potential for LVOT obstruction (Figure 5). Additionally, the open approach allows for a potential limited septal myectomy if pre-operative planning has identified a significant LVOT risk. In our experience to date, this has not been needed. The use of the guiding sutures to orient and align the valve has allowed for reproducible results. We find it an important component of the procedure, both for aligning the THV at the proper level within the annular plane, as well as for creating the proper axial alignment within the annulus to avoid canting the valve into the LVOT, and for orienting the THV commissures to subtend the LVOT. It is noted that fluoroscopy is not required nor commonly used intraoperatively with this technique. The guiding sutures also serve to further anchor the balloonexpandable THV, mitigating against migration or embolization.

Prosthetic PVL in the mitral position has been a more challenging problem in part because of the higher systolic pressures exerted on the closed mitral valve compared with the lower diastolic pressure on an aortic prosthesis.
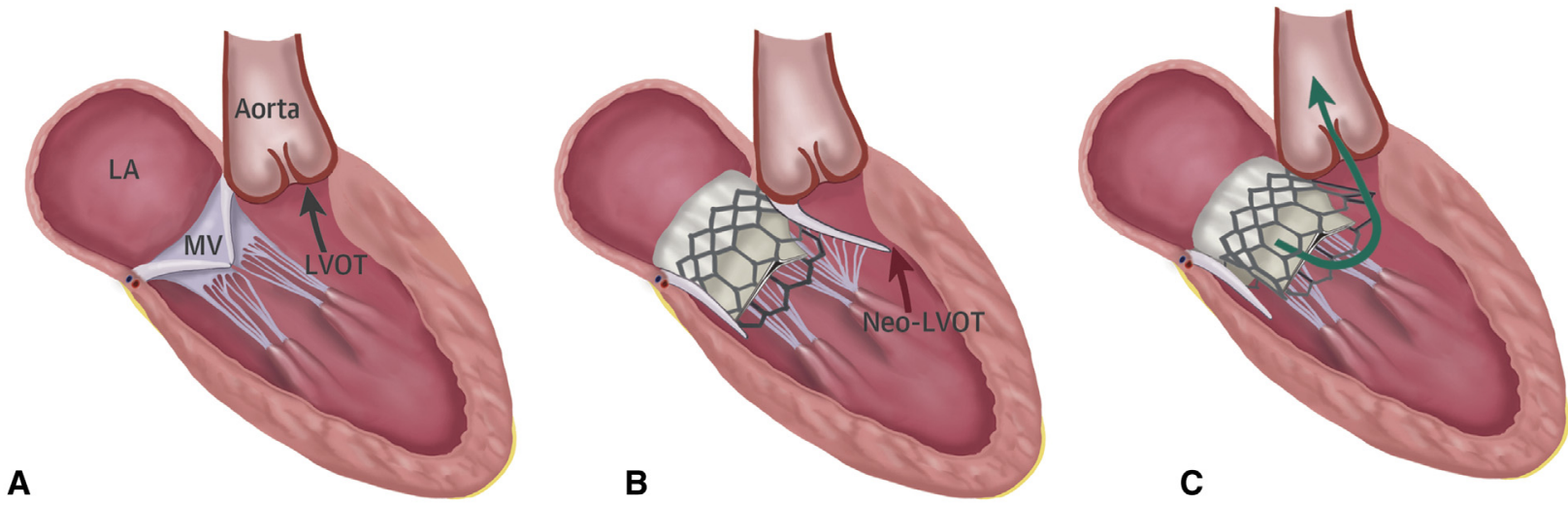

FIGURE 5. Illustration depicting (A) the normal left ventricular outflow tract (LVOT) (black arrow) and (B) the "neo-left ventricular outflow tract" (neoLVOT) (red arrow). Analysis of the neo-LVOT is critical because the anterior leaflet may cause outflow tract obstruction after placement of the transcatheter heart valve. Resection of the anterior leaflet and proper alignment of the prosthetic commissures (C) can ensure systolic flow through the open cells of the stent frame (green arrow) and reduce the risk of neo-LVOT obstruction in these circumstances. 
Earlier attempts at using transcatheter valves to replace the mitral valve in the setting of MAC either via a transseptal, transapical, or transatrial approach have been complicated by the presence of significant PVL in some cases. The manufactured sealing skirt on the valve is not bulky enough to withstand such pressures in the setting of a variegated calcified mitral annulus. We feel the addition of the felt strip around the outside of the valve has been an important advance in our ability to reduce this complication. Not all commercially available types of surgical "felt" have the same physical properties and density. The commercial product that has worked most favorably is the softer variety and is listed in Table 1. In addition, routinely "pulling" the base of the native leaflets up against the base of the THV using pledgeted sutures assists in limiting PVL.

Concomitant surgical procedures such as coronary bypass, tricuspid valve repair, and atrial fibrillation correction surgery (maze ablation) can be considered, given the surgical exposure. The relative benefits versus the added risks would need to be weighed in any given patient. Our initial cohort contained patients who were deemed at very high risk and we chose to limit the procedure to focus solely on the mitral valve. As we gained experience and confidence with the technique, we gave consideration to concomitant procedures, and our series includes 2 patients who underwent tricuspid surgery. A detailed analysis of these decisions and their outcomes is not possible within our limited experience.

The results of this technique have thus far been encouraging. Although too small a sample for meaningful comparison to other techniques and subject to operator selection bias, our experience suggests the technique described here appears to result in successful valve replacement in what historically have been surgically challenging or surgically prohibitive cases, and in patients not suitable for percutaneous transseptal or standard transapical TMVR.

\section{Study Limitations}

This study of the safety and efficacy of the described procedure is limited by the small number of patients in the present cohort and the short follow-up time as well as the limitations inherent in a nonrandomized study.

\section{CONCLUSIONS}

Successful transatrial TMVR with the Sapien 3 THV in patients with severe MAC can be safely performed despite challenging anatomy. A systematic approach to pre-procedural planning and implantation technique is necessary to achieve technical success. The description of the technique step-bystep provided herein may be useful for operators in their early experience. TMVR with the Sapien 3 THV in MAC remains off label at this time. Further studies are needed to fully evaluate the reliability and long-term outcomes of this technique. However, in this early experience, the operation described herein has been found to be reproducible and relatively easy to demonstrate and teach to others.

\section{Conflict of Interest Statement}

Dr Guerrero has received research funding from and been a proctor for Edwards Lifesciences. Dr Salinger has been a consultant for Edwards Lifesciences and Boston Scientific; and a proctor for Edwards Lifesciences. Dr Wang has been a consultant for Edwards Lifesciences, Boston Scientific, and Materialise; and is a co-inventor on a patent application assigned to Henry Ford Health System for software prediction of LVOT obstruction. Dr Sakhuja has been a consultant/ proctor for Edwards Lifesciences and Medtronic. Dr Fang has been a consultant for Edwards Lifesciences. Dr Tang has been a proctor for Edwards Lifesciences. D. Feldman has received research funding from and been a consultant for Edwards Lifesciences, Abbott, and Boston Scientific. Dr Bapat has been a consultant for and received speaker fees from Edward Lifesciences and Medtronic. Dr George has been a consultant for Edwards Lifesciences, Medtronic, and Boston Scientific. All other authors have nothing to disclose with regard to commercial support.

\section{References}

1. Fox CS, Vasan RS, Parise H, et al. Mitral annular calcification predicts cardiovascular morbidity and mortality: the Framingham Heart Study. Circulation. 2003; 107:1492-6.

2. Abramowitz Y, Jilaihawi H, Chakravarty T, Mack MJ, Makkar RR. Mitral annulus calcification. J Am Coll Cardiol. 2015;66:1934-41.

3. Carpentier AF, Pellerin M, Fuzellier JF, Relland JY. Extensive calcification of the mitral valve anulus: pathology and surgical management. J Thorac Cardiovasc Surg. 1996;111:718-29; discussion 729-30.

4. Okada Y. Surgical management of mitral annular calcification. Gen Thorac Cardiovasc Surg. 2013;61:619-25.

5. Kurazumi H, Mikamo A, Suzuki R, Hamano K. Mitral-valve replacement for a severely calcified mitral annulus: a simple and novel technique. Eur J Cardiothorac Surg. 2011;39:407-9.

6. Uchimuro T, Fukui T, Shimizu A, Takanashi S. Mitral valve surgery in patients with severe mitral annular calcification. Ann Thorac Surg. 2016;101:889-95.

7. Lafrenière-Bessi V, Cameron-Gagné M, Perron J, et al. Mitral annular calcification and mitral valve replacement: a new approach. Ann Thorac Surg. 2018;105: e55-7.

8. Salhiyyah K, Kattach H, Ashoub A, et al. Mitral valve replacement in severely calcified mitral valve annulus: a 10-year experience. Eur J Cardiothorac Surg. 2017;52:440-4.

9. Morisaki A, Kato Y, Takahashi Y, Shibata T. Mitral valve replacement with halfand-half technique for recurrent mitral paravalvular leakage. J Heart Valve Dis 2015:24:320-2.

10. Yamazaki M, Nishigawa K, Naito K, Takanashi S. Surgical treatment of massive mitral annular calcification. Eur J Cardiothorac Surg. 2016;49:1735-6.

11. Price J, Glineur D, De Kerchove L, El Khoury G. Mitral valve repair is feasible following extensive decalcification and reconstruction of the atrioventricular groove. J Heart Valve Dis. 2015;24:46-52.

12. Lad VS, Newcomb AE, Davis PJ, Leng CY. Surgical techniques for the management of the 'hostile mitral annulus.' Heart Lung Circ. 2014;23:217-23.

13. Hussain ST, Idrees J, Brozzi NA, Blackstone EH, Pettersson GB. Use of annulus washer after debridement: a new mitral valve replacement technique for patients with severe mitral annular calcification. J Thorac Cardiovasc Surg. 2013;145 1672-4.

14. Santana O, Lamelas J. Intra-atrial placement of a mitral prosthesis in a patient with severe mitral annulus calcification: a case report. Heart Surg Forum 2010;13:E25-7.

15. Goksel OS, Inan K, Tatar T, et al. Mitral valve replacement with bileaflet preservation for complex annular calcification. Heart Surg Forum. 2008;11:E1-3. 
16. Yoshikai M, Ohnishi H, Fumoto H, Itoh M, Satoh H. Mitral valve replacement for a severely calcified mitral annulus. J Card Surg. 2007:22:502-4.

17. Sirin BH, Iskesen I, Erbuyun K. An alternative method of prosthetic mitral valve implantation in the presence of extensive calcification. Acta Cardiol. 2007;62: 199-201.

18. El-Amin WO, Thomson DS. Mitral valve replacement in a severely calcified posterior annulus: a novel technique. Heart Lung Circ. 2006;15:146-7.

19. Iida H, Mochizuki Y, Matsushita Y, Mori H, Yamada Y, Miyoshi S. A valve replacement technique for heavily calcified mitral valve and annulus. J Heart Valve Dis. 2005;14:209-11.

20. Okamoto H, Tamenishi A, Itoh Y, Niimi T. Mitral annular reconstruction with anterior leaflet flip-over in concomitant surgery for mitral regurgitation associated with extensive posterior annular calcification and hypertrophic obstructive cardiomyopathy. Jpn J Thorac Cardiovasc Surg. 2004;52:104-6.

21. Feindel CM, Tufail Z, David TE, Ivanov J, Armstrong S. Mitral valve surgery in patients with extensive calcification of the mitral annulus. J Thorac Cardiovasc Surg. 2003;126:777-82.

22. Baumgartner FJ, Pandya A, Omari BO, Turner C, Milliken JC, Robertson JM. Ultrasonic debridement of mitral calcification. J Card Surg. 1997;12:240-2.

23. Vander Salm TJ, Perras M. As originally published in 1989: mitral annular calcification: a new technique for valve replacement. Updated in 1997. Ann Thorac Surg. 1997;63:1819-20.

24. Ruvolo G, Speziale G, Voci P, Marino B. "Patch-glue" annular reconstruction for mitral valve replacement in severely calcified mitral annulus. Ann Thorac Surg. 1997;63:570-1.

25. Said SM, Schaff HV. An alternate approach to valve replacement in patients with mitral stenosis and severely calcified annulus. J Thorac Cardiovasc Surg. 2014; 147:e76-8.

26. El Sabbagh A, Eleid MF, Foley TA, et al. Direct transatrial implantation of balloon-expandable valve for mitral stenosis with severe annular calcifications: early experience and lessons learned. Eur J Cardiothorac Surg. 2018;53:162-9.

27. Ghosh-Dastidar M, Bapat V. Transcatheter valve implantation in mitral annular calcification during open surgery: extended collar technique. Ann Thorac Surg. 2017; 104:e303-5.

28. Polomsky M, Koulogiannis KP, Kipperman RM, et al. Mitral valve replacement with Sapien 3 transcatheter valve in severe mitral annular calcification. Ann Thorac Surg. 2017; 103:e57-9.

29. Koeckert MS, Loulmet DF, Williams MR, Neuburger PJ, Grossi EA. Robotic transcatheter mitral valve replacement using the Sapien XT in the setting of severe mitral annular calcification. J Card Surg. 2016;31:303-5.

30. El-Eshmawi A, Love B, Bhatt HV, Pawale A, Boateng P, Adams DH. Direct access implantation of a Melody valve in native mitral valve: a hybrid approach in the presence of extensive mitral annular calcification. Ann Thorac Surg. 2015:99:1085.

31. Lim ZY, Boix R, Prendergast B, et al. First reported case of transcatheter mitral valve implantation in mitral annular calcification with a fully repositionable and self-expanding valve. Circ Cardiovasc Interv. 2015;8:e003031.

32. Ferrari E, Niclauss L, Locca D, Marcucci C. On-pump fibrillating heart mitral valve replacement with the SAPIEN ${ }^{\mathrm{TM}}$ XT transcatheter heart valve. Eur J Cardiothorac Surg. 2014:45:749-51.

33. Carrel T, Wenaweser P, Reineke S, et al. Worldwide first surgical implantation of a transcatheter valved stent in mitral position. Cardiovasc Med. 2012;15:202-5.

34. Astarci P, Glineur D, De Kerchove L, El Khoury G. Transcatheter valve used in a bailout technique during complicated open mitral valve surgery. Interact Cardiovasc Thorac Surg. 2013;17:745-7.
35. Murashita T, Suri RM, Daly RC. Sapien XT transcatheter mitral valve replacement under direct vision in the setting of significant mitral annular calcification. Ann Thorac Surg. 2016;101:1171-4.

36. Guerrero M, Dvir D, Himbert D, et al. Transcatheter mitral valve replacement in native mitral valve disease with severe mitral annular calcification: results from the first multicenter global registry. J Am Coll Cardiol Intv. 2016;9: 1361-71.

37. Verma D, Pershad A, Morse M, et al. TCT-465: a US multicenter registry for transcatheter mitral valve replacement using balloon expandable valve in patients who are at prohibitive risk for surgery (abstr). J Am Coll Cardiol. 2017;70 Suppl B:B192.

38. Guerrero M. 30-Day outcomes of transcatheter mitral valve replacement in patient with severe mitral valve disease secondary to mitral annular calcification of failed annuloplasty rings. Paper presented at: Transcatheter Cardiovascular Therapeutics Scientific Symposium; 2017.

39. Guerrero M, Salinger M, Pursnani A, et al. Transseptal transcatheter mitral valvein-valve: a step by step guide from preprocedural planning to postprocedural care. Catheter Cardiovasc Interv. 2017 May 30 [E-pub ahead of print].

40. Wang DD, Eng M, Greenbaum A, et al. Predicting LVOT obstruction after TMVR. J Am Coll Cardiol Img. 2016;9:1349-52.

41. El Sabbagh A, Eleid MF, Matsumoto JM, et al. Three-dimensional prototyping for procedural simulation of transcatheter mitral valve replacement in patients with mitral annular calcification. Catheter Cardiovasc Interv. 2018 Jan 23 [Epub ahead of print].

42. Achenbach S, Delgado V, Hausleiter J, Schoenhagen P, Min JK, Leipsic JA SCCT expert consensus document on computed tomography imaging before transcatheter aortic valve implantation (TAVI)/transcatheter aortic valve replacement (TAVR). J Cardiovasc Comput Tomogr. 2012;6:366-80.

43. Narang A, Guerrero M, Feldman T, Pursnani A. Computed tomography assessment for transcatheter mitral valve interventions. J Cardiovasc Surg (Torino). 2016;57:360-71.

44. Wang DD, Eng MH, Greenbaum AB, et al. Validating a prediction modeling tool for left ventricular outflow tract (LVOT) obstruction after transcatheter mitral valve replacement (TMVR). Catheter Cardiovasc Interv. 2017 Dec 11 [E-pub ahead of print].

45. Wang D, Eng M, Greenbaum A, Guerrero M, O’Neill W. TCT-626: Validating a prediction modeling tool for LVOT obstruction after transcatheter mitral valve replacement. J Am Coll Cardiol. 2016;68 Suppl B:B254-5.

46. Russell H, Guerrero M. Available at: https://drive.google.com/file/d/0Bw1IiF9q9K2TlZzTmlMalI5SDg/view?usp=sharing. Accessed August 6, 2018

47. Capretti G, Urena M, Himbert D, et al. Valve thrombosis after transcatheter mitral valve replacement. J Am Coll Cardiol. 2016;68:1814-5.

48. Eng MH, Greenbaum A, Wang DD, et al. Thrombotic valvular dysfunction with transcatheter mitral interventions for postsurgical failures. Catheter Cardiovasc Interv. 2017;90:321-8.

49. Stone GW, Adams DH, Abraham WT, et al. Clinical trial design principles and endpoint definitions for transcatheter mitral valve repair and replacement: part 2: endpoint definitions: a consensus document from the mitral valve academic research consortium. J Am Coll Cardiol. 2015;66:308-21.

Key Words: mitral annular calcification, surgery, valve replacement 